\title{
$\mathrm{X}$-ray diffraction and spectroscopy study of nano- $\mathrm{Eu}_{2} \mathrm{O}_{3}$ structural
}

\section{transformation under high pressure}

\author{
Zhenhai Yu, ${ }^{1}$ Qinglin Wang ${ }^{1,2}$, Yanzhang Ma ${ }^{1,3}$, Lin Wang ${ }^{1 *}$, \\ ${ }^{1}$ Center for High Pressure Science and Technology Advanced Research, Shanghai, 201203, China \\ ${ }^{2}$ Shandong Key Laboratory of Optical Communication Science and Technology, School of Physics \\ Science \& Information Technology, Liaocheng University, Liaocheng 252059, China \\ ${ }^{3}$ Department of Mechanical Engineering, Texas Tech University, Lubbock, Texas 79409, United States \\ of America \\ *Corresponding Author E-mail: wanglin@hpstar.ac.cn (Dr. Lin Wang)
}

\section{Keywords:}

Rare Earth Sesquioxides; Nanocrystalline Materials; High pressure; Structural phase transition; 


\section{Abstract}

Nanoscale materials exhibit properties that are quite distinct from those of bulk materials because of their size restricted nature. Here, we investigated the high-pressure structural stability of cubic (C-type) nano- $\mathrm{Eu}_{2} \mathrm{O}_{3}$ using in situ synchrotron X-ray diffraction (XRD), Raman and luminescence spectroscopy, and impedance spectra techniques. Our high-pressure XRD experimental results revealed a pressure-induced structural phase transition in nano- $\mathrm{Eu}_{2} \mathrm{O}_{3}$ from the $\mathrm{C}$-type phase (space group: $I a$ 3) to a hexagonal phase (A-type, space group: $P-3 m 1)$. Our reported transition pressure $(9.3 \mathrm{GPa})$ in nano- $\mathrm{Eu}_{2} \mathrm{O}_{3}$ is higher than that of the corresponding bulk- $\mathrm{Eu}_{2} \mathrm{O}_{3}(5.0 \mathrm{GPa})$, which is contrary to the preceding reported experimental result. After pressure release, the A-type phase of $\mathrm{Eu}_{2} \mathrm{O}_{3}$ transforms into a new monoclinic phase (B-type, space group: $\mathrm{C2} / \mathrm{m}$ ). Compared with bulk- $\mathrm{Eu}_{2} \mathrm{O}_{3}, \mathrm{C}$-type and Atype nano- $\mathrm{Eu}_{2} \mathrm{O}_{3}$ exhibits a larger bulk modulus. Our Raman and luminescence findings and XRD data provide consistent evidence of a pressure-induced structural phase transition in nano- $\mathrm{Eu}_{2} \mathrm{O}_{3}$. To our knowledge, we have performed the first high-pressure impedance spectra investigation on nano-Eu $\mathrm{U}_{2} \mathrm{O}_{3}$ to examine the effect of the structural phase transition on its transport properties. We propose that the resistance inflection exhibited at $\sim 12 \mathrm{GPa}$ results from the phase boundary between the C-type and A-type phases. Besides, we summarized and discussed the structural evolution process by the phase diagram of lanthanide sesquioxides $\left(\operatorname{Ln}_{2} \mathrm{O}_{3}\right)$ under high pressure.

\section{Introduction}

Lanthanide sesquioxides $\left(\operatorname{Ln}_{2} \mathrm{O}_{3}, \mathrm{Ln}=\right.$ lanthanide $)$ possessing partially filled $f$ shells, have attracted much attention during the past decades due to their various physical properties and wide applications [1]. Some of these oxides have potential application as the nuclear waste host materials $[2,3]$. Therefore, it is crucial to understand their structural stabilities at extreme conditions of pressure and temperature. From the perspective of crystallography, $\mathrm{Ln}_{2} \mathrm{O}_{3}$ mainly crystallize into three types of 
crystal polymorphisms at ambient conditions. For example, $\mathrm{Ln}_{2} \mathrm{O}_{3}$ with larger $\mathrm{La}, \mathrm{Ce}, \mathrm{Pr}$ and $\mathrm{Nd}$ cations crystallize into a hexagonal structure (Space group: $P-3 m 1$, hereafter A-type). The medium $\mathrm{Sm}$, Eu or Gd cations containing $\mathrm{Ln}_{2} \mathrm{O}_{3}$ mainly present a monoclinic (Space group: C2/m, hereafter B-type) or cubic (Space group: Ia-3, hereafter C-type) structure. The schematic crystal models (C-, A-, and B-types) are shown in Fig. 1 (a). While the smaller cations (from $\mathrm{Tb}$ to $\mathrm{Lu}$ ) containing $\mathrm{Ln}_{2} \mathrm{O}_{3}$ mainly crystallize into a C-type structure. Nanoscale materials exhibit properties that are quite distinct from those of bulk materials because of their size restricted nature. The difference in structural evolution behavior of nanoscale materials compared to bulk materials was mostly believed to be a result of the competition between the specific surface area, grain boundary and the internal energy differences. To compare the similarities and differences between bulk and nanoscale materials, here we have studied structural phase transition in nano- $\mathrm{Eu}_{2} \mathrm{O}_{3}$ using a combined high pressure synchrotron $\mathrm{X}$ ray diffraction (XRD) and spectroscopy techniques. Furthermore, the phase diagram of the structural evolution process for $\mathrm{Ln}_{2} \mathrm{O}_{3}$ under high pressure was summarized and discussed.

The temperature dependence of the lanthanide sesquioxides polymorphisms has been thoroughly studied for fundamental and applied reasons [4]. In addition, external pressure is a well-known powerful method to decrease the interatomic distance and increase the density of materials, eventually changing their crystal polymorphisms. At ambient conditions, the density sequences of these three typical polymorphisms for $\mathrm{A}-, \mathrm{B}-$ and $\mathrm{C}-$ types of $\mathrm{Ln}_{2} \mathrm{O}_{3}$ are $\rho_{\mathrm{A}}>\rho_{\mathrm{B}}>\rho_{\mathrm{C}}$. Therefore, it is expected that the structural phase transition sequences in $\mathrm{Ln}_{2} \mathrm{O}_{3}$ under high pressure will follow the order $\mathrm{C} \rightarrow \mathrm{B}$ $\rightarrow$ A. Previous in-situ high pressure experiments on these $\mathrm{Ln}_{2} \mathrm{O}_{3}$ have confirmed this speculation, such as the phase transition of $\mathrm{B} \rightarrow \mathrm{A}$ in $\mathrm{Sm}_{2} \mathrm{O}_{3}$ [5], the phase transition of $\mathrm{C} \rightarrow \mathrm{A}$ in $\mathrm{Gd}_{2} \mathrm{O}_{3}$ [6] and the phase transition of $\mathrm{C} \rightarrow \mathrm{B} \rightarrow \mathrm{A}$ in $\mathrm{Dy}_{2} \mathrm{O}_{3}$ [7]. Sc and $\mathrm{Y}$ are considered as rare earth elements because they tend to occur in the same ore deposits as the lanthanides and exhibit similar chemical properties. Recently, $\mathrm{Gd}_{2} \mathrm{~S}_{3}$-type phase (Space group: Pnma) was discovered in $\mathrm{Sc}_{2} \mathrm{O}_{3}$ and $\mathrm{Y}_{2} \mathrm{O}_{3}$ under high 
pressure and high temperatures [8,9]. This also evoked further studies on the structural phase transitions in rare earth sesquioxides under extreme conditions.

$\mathrm{Eu}_{2} \mathrm{O}_{3}$, which is one of the most important rare-earth oxides, has been extensively utilized as high performance luminescent devices, neutron absorbers, catalysts etc. based on its unique optical and chemical characteristics arising from its $4 f$ electrons [10]. Due to these interesting applications, it is crucial to understand the characteristics of its different phases under external conditions. The pressureinduced phase transitions in $\mathrm{Eu}_{2} \mathrm{O}_{3}$ have previously been studied using XRD, Raman spectroscopy and other techniques. However, the phase transition in $\mathrm{Eu}_{2} \mathrm{O}_{3}$ from the reported results was inconsistent. A pressure-induced $\mathrm{C} \rightarrow \mathrm{B}$ structural phase transition in bulk- $\mathrm{Eu}_{2} \mathrm{O}_{3}$ was reported by Chen et al. by the luminescence spectra of Eu ions [11]. Later, a pressure-induced structural phase transition from a B- to A-type in bulk- $\mathrm{Eu}_{2} \mathrm{O}_{3}$ was discovered around 4.7 GPa using energy dispersive XRD technique [12]. The high-pressure structural evolution of bulk- $\mathrm{Eu}_{2} \mathrm{O}_{3}$ was investigated by Jiang et al. using an angle dispersive XRD (AD-XRD) technique. Their experimental results showed that bulk- $\mathrm{Eu}_{2} \mathrm{O}_{3}$ undergoes a C-type $\rightarrow$ A-type structural phase transition with a coexist pressure range of 5.0 GPa $~ 13.0 \mathrm{GPa}$ [13]. From these preceding investigations, there are still significant discrepancies between the pressureinduced structural phase transition sequence in bulk- $\mathrm{Eu}_{2} \mathrm{O}_{3}$ by different authors. Here, we extended the investigation on pressure-induced structural phase transition in nano- $\mathrm{Eu}_{2} \mathrm{O}_{3}$. Our motivation was to discover if nano- $\mathrm{Eu}_{2} \mathrm{O}_{3}$ presents a different phase transition sequence from bulk- $\mathrm{Eu}_{2} \mathrm{O}_{3}$ under high pressure.

Comparative investigations on pressure-induced structural phase transition in bulk- and nanoscale-materials have attracted extensive attention during the past decades $[14,15]$. To the best of our knowledge, nano-materials with a smaller particle size will exhibit higher transition pressures, such as the particle size dependence of the phase transition pressure investigated in $\mathrm{Y}_{2} \mathrm{O}_{3}$ [16]. Smaller particle sizes resulting in a significant reduction of the phase transition pressure were only reported in 
$\mathrm{CeO}_{2}, \mathrm{TiO}_{2}$ and $\gamma-\mathrm{Fe}_{2} \mathrm{O}_{3}$ [17-19]. The pressure-induced structural phase transition in nano- $\mathrm{Eu}_{2} \mathrm{O}_{3}$ was studied by Dilawar et al. using a laboratory rotating anode generator X-ray source [20]. The authors suggested that nano- $\mathrm{Eu}_{2} \mathrm{O}_{3}$ transforms from a $\mathrm{C}$-type to $\mathrm{B}$-type phase around $2.0 \mathrm{GPa}$, which is remarkably lower than the phase transition point of 5.0 GPa that occurs in the corresponding bulk$\mathrm{Eu}_{2} \mathrm{O}_{3}$. For the transition pressure reported in Ref. [20], there is still no other literature to refer to. The purpose of this paper is to report our detailed results of the pressure-induced transition in nano- $\mathrm{Eu}_{2} \mathrm{O}_{3}$ and compare our results with preceding investigations on these structural transitions. The morphology of the present sample was investigated with transmission electron microscopy (TEM). $\operatorname{Ln}_{2} \mathrm{O}_{3}$ compounds are important materials for different technological application requiring specific optical, magnetic, and electronic properties. This makes it crucial to understand the transport behavior of $\operatorname{Ln}_{2} \mathrm{O}_{3}$ at different conditions. We have performed the first high-pressure impedance spectra investigation on nano- $\mathrm{Eu}_{2} \mathrm{O}_{3}$, to examine the effect of the structural phase transition on its transport properties. It is remarkable to note that the resistance exhibits inflection around of the C-type $\rightarrow$ A-type transition pressure.

\section{Experimental methods}

\subsection{High-pressure synchrotron angle dispersive XRD}

We performed our high-pressure synchrotron XRD experiment using a symmetric diamond anvil cell (DAC) with a $300 \mu \mathrm{m}$ culet. T301 stainless steel with a thickness of $310 \mu \mathrm{m}$ and a pre-indented thickness of $20 \mu \mathrm{m}$ served as our gasket. The $120 \mu \mathrm{m}$ diameter sample chamber was filled with a mixture of the nano- $\mathrm{Eu}_{2} \mathrm{O}_{3}$ compound, a ruby chip, and silicone oil as a pressure-transmitting medium. Angle dispersive powder XRD patterns were taken with a Mar3450 detector using synchrotron radiation beams monochromatized to a wavelength of $0.3875 \AA$ at the 16 BM-D beamline of the Advanced Photon Source (APS), Argonne National Laboratory (ANL). The two-dimensional (2-D) 
image plate patterns were converted to one-dimensional versus intensity data using the Fit2D software package [21]. The experimental pressures were determined by the pressure-induced fluorescence ruby shift [22].

\subsection{Raman and photoluminescence (PL) spectra under high-pressure}

We carried out our Raman spectroscopy investigation on nano- $\mathrm{Eu}_{2} \mathrm{O}_{3}$ under high pressure using a commercial Renishaw Raman spectroscopy system in the backscattering configuration excited with a $\mathrm{He} / \mathrm{Ne}$ laser $(\lambda=632.8 \mathrm{~nm})$ and a spectra resolution of around $1 \mathrm{~cm}^{-1}$. We used a methanol and ethanol mixture as our pressure-transmitting medium. High-pressure PL spectra were also performed using the commercial Renishaw Raman spectroscopy system and excitation sources $532 \mathrm{~nm}$. The pressure of the sample (both for Raman and luminescence measurements) was measured using well established ruby fluorescence techniques [22].

\subsection{High-pressure impedance spectroscopy properties measurement}

A symmetric DAC with an anvil culet of $400 \mu \mathrm{m}$ in diameter was utilized to generate high pressure. A two-electrode (Pt electrodes) configuration was used for in-situ electrical impedance spectroscopy measurement on the DAC under high pressure. The manufacturing process of the microcircuit was similar to that described in our previous works [23]. We did not use a pressure-transmitting medium to ensure that the impedance spectroscopy reflected the sample information. A ruby ball was used as the pressure calibrator by using the wavelength shift of its R1 line [22]. The impedance spectra were measured by a Solartron 1260 impedance analyzer equipped with Solartron 1296 dielectric interface. A 3.0 $\mathrm{V}$ sine signal was input into the sample, and its frequency ranged from $10^{-2}$ to $10^{7} \mathrm{~Hz}$.

\section{Results and discussion}

\subsection{The morphology of nano- $\mathrm{Eu}_{2} \mathrm{O}_{3}$}


The nano- $\mathrm{Eu}_{2} \mathrm{O}_{3}$ sample used in the present study was commercially purchased from pearl river rare earth smelter. The phase purity of sample was confirmed by using XRD measurement. The sample was used for high-pressure measurements without further process. First, the morphology of nano- $\mathrm{Eu}_{2} \mathrm{O}_{3}$ was analyzed using TEM. The TEM image of the nano- $\mathrm{Eu}_{2} \mathrm{O}_{3}$ is shown in Fig. 1 (b). The sample and the background present an apparent contrast. The grain size of the nano- $\mathrm{Eu}_{2} \mathrm{O}_{3}$ sample was analyzed, and the size of the grain ranges from 6 to $18 \mathrm{~nm}$. The diameters of the nano- $\mathrm{Eu}_{2} \mathrm{O}_{3}$ crystallite size were also estimated using the Debye-Scherrer formula $d=0.89 \lambda /(\beta \cos \theta)$, where $\lambda$ is the $\mathrm{X}$-ray wavelength, $\beta$ is the width of the diffraction peak at full width at half maximum (FWHM) and $\theta$ is the Bragg diffraction angle. We deduced that the nano- $\mathrm{Eu}_{2} \mathrm{O}_{3}$ nanoparticles have an average crystallite size of $\sim$ $18 \mathrm{~nm}$ by analysis of the XRD peak at $2 \theta=7.07^{\circ}(\mathrm{hkl}$ : (222)), as shown in Fig. 2 (a).

\subsection{High pressure angle dispersive $\mathrm{XRD}$ patterns of nano-Eu $\mathbf{u}_{2} \mathrm{O}_{3}$}

Fig. 2 (a) demonstrates the selected AD-XRD patterns of nano- $\mathrm{Eu}_{2} \mathrm{O}_{3}$ under various pressures during compression. As the pressure gradually increases, the diffraction peaks of the C-type phase shift steadily to a higher angle. Apart from the peak shift, the diffraction peaks do not undergo any significant variation up to the pressure of $9.3 \mathrm{GPa}$. Above $11.2 \mathrm{GPa}$, the appearance of new diffraction peaks at $2 \theta=6.8^{\circ}\left(\mathrm{hkl}\right.$ : (100)) and $7.9^{\circ}(\mathrm{hkl}$ : (101)) from C-type ((222) and (312)) indicates a pressureinduced structural phase transition. As the pressure further increases, the C-type phase disappears and the peaks corresponding to the A-type phase become progressively more defined. XRD patterns of nano- $\mathrm{Eu}_{2} \mathrm{O}_{3}$ were analyzed with Rietveld refinement using the GSAS program package [24] with the user interface EXPGUI [25]. We obtained the lattice parameter value for C-type ( $a=10.884 \AA$ at 0 GPa) and A-type phases ( $a=b=3.685 \AA, c=5.616 \AA$ at $17.6 \mathrm{GPa})$ by Rietveld refinement. The pressure-induced structural phase transition (C-type $\rightarrow$ A-type) in nano- $\mathrm{Eu}_{2} \mathrm{O}_{3}$ results into the appearance of a new vibrational mode and variation of the energy band structure, which we analyzed in

the subsequent Raman and luminescence measurements. Further pressure increase up to $43.0 \mathrm{GPa}$ did 
not lead to any new diffraction peaks but peaks broadening, which indicates that the A-type phase could stably exist up to the highest pressure.

It should be emphasized that the AD-XRD data obtain in Ref. [20] are seriously affected by the gasket signal because of the large beam size $(100 \mu \mathrm{m})$ of the laboratory $\mathrm{X}$-ray radiation $(\lambda=0.71069$ A) which simultaneously impinges the sample and gasket. Furthermore, Dilawar et al. in Ref. [20] suggested that nano- $\mathrm{Eu}_{2} \mathrm{O}_{3}$ exhibits a pressure-induced structural phase transition according to the intensity change of a diffraction peak at $2 \theta=12.965^{\circ}$. However, the weakening diffraction patterns suffered from several factors such as (i) the decreasing depth of the sample; (ii) the quasihydrostatic or nonhydrostatic pressure environment; and (iii) strong crystallographic distortion. Therefore, we suggested that the phase transition drawn from XRD data should be complemented with other characterized technique such as Raman spectroscopy. N. Dilawar et al. [20] used a 4:1 mixture of methanol-ethanol, gold, and ruby as the pressure transmitting medium, the pressure marker in XRD and Raman measurement, respectively. The starting sample used in Dilawar's experiment was found to be predominantly cubic type $\mathrm{Eu}_{2} \mathrm{O}_{3}$ or in $\mathrm{Eu}^{3+}$ state with a trace of $\mathrm{Eu}^{2+}$ (non-stoichiometric $\mathrm{Eu}_{1-\mathrm{x}} \mathrm{O}$ phase). We speculated that the above mentioned may cause the difference between Dilawar et al. and present work.

In order to check whether or not the pressure-induced structural phase transition in nano- $\mathrm{Eu}_{2} \mathrm{O}_{3}$ is reversible, we also collected the XRD patterns during pressure release. We found that A-type $\mathrm{Eu}_{2} \mathrm{O}_{3}$ transforms into B-type after pressure release to ambient pressure, as shown in Fig. 2 (b). This indicates that the pressure-induced structural phase transition in nano- $\mathrm{Eu}_{2} \mathrm{O}_{3}$ is irreversible. The process of structural phase transition depends on several factors such as energy, kinetic barrier, nucleation, phase transition path etc.; thus, A-type may transform into C- or B-type as the pressure decreases. From an energy landscape perspective, $\mathrm{B}$-type $\mathrm{Eu}_{2} \mathrm{O}_{3}$ has higher energy than $\mathrm{C}$-type under ambient pressure. However, our experimental results show that nano- $\mathrm{Eu}_{2} \mathrm{O}_{3}$ undergoes a structural phase transition of 
A-type $\rightarrow$ B-type as pressure releases to ambient pressure. This phenomenon could stem from the lower kinetic barrier of the A-type $\rightarrow$ B-type than the A-type $\rightarrow$ C-type phase transition, which may originate from the reconstructive nature of the A-type $\rightarrow$ C-type phase transition.

Preceding high-pressure $\mathrm{XRD}$ studies on bulk- $\mathrm{Eu}_{2} \mathrm{O}_{3}$ indicated that the $\mathrm{C}$-type $\rightarrow \mathrm{A}$-type phase transition begins at 5.0 GPa [13], which is lower than our present transition pressure of 9.3 GPa. Here, our experimental results are consistent with the general model for most nanomaterials (including oxides and sulfides), which exhibit higher transition pressures with a decrease in particle size [26,27]. We suggest that the reduction of particle size dramatically enhances the structure stability of $\mathrm{Eu}_{2} \mathrm{O}_{3}$. Fig. 3 (a) exhibits the pressure dependence of the normalized lattice parameters of the C- and A-type phases for nano- $\mathrm{Eu}_{2} \mathrm{O}_{3}$. We found that the axial compressibilities of the A-type phase are remarkably anisotropic, which was characterized by kappa parameter $\kappa\left(\kappa=\left(a_{0}-a_{p}\right) / a_{0}, a_{0}\right.$ is lattice parameter at ambient pressure, while $a_{p}$ is lattice parameter under high pressure). The compressibility along $a$-axis is about one order of magnitude smaller than along the $c$-axis. The axial compressibility of the $\mathrm{C}$-type phase is similar to the $c$-axis compressibility of the A-type phase. The axial ratio $(c / a)$ of the A-type nano- $\mathrm{Eu}_{2} \mathrm{O}_{3}$, which decreases with the increasing pressure is illustrated as a function of pressure in the inset of Fig. 3 (a). These results are in good qualitative agreement with the normalized lattice parameter data.

Fig. 3 (b) illustrates the pressure-dependent unit cell volumes of the C-type and A-type phases of $\mathrm{Eu}_{2} \mathrm{O}_{3}$, where the solid lines are the fitting results of the $\mathrm{C}$-type and $\mathrm{A}$-type phases [28]. The equation of state (EOS) parameters $V_{0}$ (unit cell volume at ambient pressure), $B_{0}$ (isothermal bulk modulus), and $B_{0}$ ' (pressure derivative of $B_{0}$ ) were determined by a $2^{\text {nd }}$ order Birch-Murnaghan equation fitting [29]. With $B_{0}$ ' fixed as 4 , we obtained the ambient pressure isothermal bulk modulus of $B_{0}=178(5)$ and 229 (2) GPa for the C-type and A-type phases, respectively. The bulk modulus of C-type bulk-Eu $\mathrm{O}_{3}$ is $~$ 
$145 \mathrm{GPa}$ according to previous high pressure XRD investigations and theoretical simulations $[13,30]$. The $B_{0}$ of nano- $\mathrm{Eu}_{2} \mathrm{O}_{3}$ is $\sim 20 \%$ larger than its bulk counterpart. This phenomenon may be due to the higher surface to volume ratio of nano- $\mathrm{Eu}_{2} \mathrm{O}_{3}$ than bulk- $\mathrm{Eu}_{2} \mathrm{O}_{3}$. The $\mathrm{C}$-type $\rightarrow$ A-type phase transition is accompanied by a drastic decrease of about $12.1 \%$ in its volume. The C-type $\rightarrow$ A-type phase transformation is reconstructive and the volume change should be large. Zinkevich [1] summarized that the relative volume changes of the C-type $\rightarrow$ A-type phase transitions for most $\mathrm{Ln}_{2} \mathrm{O}_{3}$ were about $8 \%$.

\subsection{Raman and photoluminescence (PL) spectra of nano-E $\mathbf{u}_{2} \mathrm{O}_{3}$ under high-pressure}

To further verify our proposed structural phase transition sequence of nano- $\mathrm{Eu}_{2} \mathrm{O}_{3}$ under high pressure, we employed Raman scattering spectroscopy to characterize its pressure-induced structural phase transition. Our nano- $\mathrm{Eu}_{2} \mathrm{O}_{3}$ sample crystallizes into a cubic structure with space group $I a-3$ at ambient conditions from our XRD data analysis. The unit cell of the C-type phase has a bixbyite structure, which contains 16 molecules of $\mathrm{Eu}_{2} \mathrm{O}_{3}$. Twenty-two Raman modes $\left(4 \mathrm{~A}_{\mathrm{g}}+4 \mathrm{E}_{\mathrm{g}}+14 \mathrm{~F}_{\mathrm{g}}\right)$ have been predicted for the $\mathrm{C}$-type $\mathrm{Eu}_{2} \mathrm{O}_{3}$ according to group theory analysis [31]. At ambient conditions, only 12 Raman peaks at $\left(94,109,119,134,145,175,289,337,385,425,559\right.$, and $\left.671 \mathrm{~cm}^{-1}\right)$ for the cubic phase were observed clearly. It is clear from Fig. 4 that the strongest Raman peak for nano- $\mathrm{Eu}_{2} \mathrm{O}_{3}$ is observed at $337 \mathrm{~cm}^{-1}$ at ambient conditions. As reported, the sample shows characteristic peaks that correspond to the cubic phase of bulk- $\mathrm{Eu}_{2} \mathrm{O}_{3}$. The most prominent peak assigned as the $\mathrm{F}_{\mathrm{g}}$ band occurs at $337 \mathrm{~cm}^{-1}$. Our experimental results are in good agreement with previous reported results [32]. A-type $\mathrm{Eu}_{2} \mathrm{O}_{3}$ is hexagonal (space group: $P-3 m 1$ ), and contains one molecule per unit cell. B-type $\mathrm{Eu}_{2} \mathrm{O}_{3}$ is monoclinic (space group: $C 2 / m$ ), and contains six molecules per unit cell. Our group theory analysis yields 21 Raman modes: $14 \mathrm{~A}_{\mathrm{g}}+7 \mathrm{~B}_{\mathrm{g}}$ for the $\mathrm{B}$-type phase, and $\mathrm{A}$-type phase shows four Raman modes: $2 \mathrm{~A}_{1 \mathrm{~g}}+2 \mathrm{E}_{\mathrm{g}}$.

With increasing pressure, all Raman modes show continuous shifts to higher wavenumbers, indicating $\mathrm{Eu}-\mathrm{O}$ bonding contraction under high pressure. However, as the pressure further increases, 
only the strong Raman modes are clearly observable. Above $9.7 \mathrm{GPa}$, the relative intensity of the Raman peaks exhibit a remarkable variation compared to ambient pressure, and the Raman peaks become broader and new Raman peaks appeared. Considering our XRD, Raman data and literature results [6,33], we think a structural change occurs above this pressure point. Dilawa et al. [20] suggested that the C-type $\rightarrow$ A-type phase transition occurred at $2.94 \sim 8.08$ GPa by Raman spectra. The Raman signal of the A-type phase of nano- $\mathrm{Eu}_{2} \mathrm{O}_{3}$ is very weak and only a broad peak is observed at frequency $\sim 500 \mathrm{~cm}^{-1}$. As mentioned above, the pressure-induced structural phase transition in nano$\mathrm{Eu}_{2} \mathrm{O}_{3}$ was irreversible according to the XRD results. Therefore, we also collected the Raman spectrum of $\mathrm{Eu}_{2} \mathrm{O}_{3}$ after decompressing the sample to ambient conditions as shown in Fig. 4. The nano- $\mathrm{Eu}_{2} \mathrm{O}_{3}$ quenched from 43.0 GPa shows a completely different Raman spectrum from the starting sample. As compared with the reported Raman spectrum of other rare-earth sesquioxides, the quenched nano$\mathrm{Eu}_{2} \mathrm{O}_{3}$ has a B-type structure. The observed pressure-induced structural phase transition using Raman spectrum is in good agreement with the XRD experimental observations.

$\mathrm{Eu}^{3+}$ is often studied as a useful structural probe, which is sensitive to the surrounding crystal environment. Hence, we used PL measurement as a unique probe to investigate the effect of the nano$\mathrm{Eu}_{2} \mathrm{O}_{3}$ phase transition under high pressure. The $\mathrm{C}$-type $\mathrm{Eu}_{2} \mathrm{O}_{3}$ crystal structures possess Ia-3 symmetry at ambient conditions with a large band gap $(\sim 4.4 \mathrm{eV})[34]$. There are two $\mathrm{Eu}^{3+}$ sites (two Wyckoff position 8(b) and $24(\mathrm{~d})$ ), and these sites possess C2 and S6 symmetry. The sites occur in a 3:1 ratio in the unit cell and the $\mathrm{C} 2$ site is more abundant [35], and therefore, the ${ }^{5} \mathrm{D}_{0}-{ }^{7} \mathrm{~F}_{2}$ transition is prominent. Our C-type phase of nano- $\mathrm{Eu}_{2} \mathrm{O}_{3}$ under an excitation wavelength (532 nm) displays characteristics sharp peaks in the 550-720 nm region associated with the ${ }^{5} \mathrm{D}_{0}{ }^{-}{ }^{7} \mathrm{~F}_{\mathrm{J}},(\mathrm{J}=0,1,2,3)$. Fig. 5 (a) shows selected luminescence spectra obtained from $\mathrm{C}$-type nano- $\mathrm{Eu}_{2} \mathrm{O}_{3}$ under various pressures up to $30 \mathrm{GPa}$. The luminescence located around $572 \mathrm{~nm}$ originates from the diamond and is labeled with dashed lines. Furthermore the luminescence peak of the diamond shows a feeble pressure shift due to 
the large bulk modulus ( $445 \mathrm{GPa})$ and band gap $(5.5 \mathrm{eV})$ [36]. The luminescence spectra obtained under ambient pressure and up to $12.1 \mathrm{GPa}$ exhibit features of C-type symmetry. The luminescence spectra experienced minor red shift as the pressure increases from ambient pressure to $12.1 \mathrm{GPa}$. The luminescence spectrum obtained at $12.1 \mathrm{GPa}$ differs from that at ambient pressure in the compression process. Furthermore, the relative loss of intensity in band I with respect to band II is further evidence that the structural phase transition of C-type $\rightarrow$ A-type has occurred as shown in Fig. 5 (b). It is known that the luminescent properties of inorganic materials can be greatly affected by tailoring their crystallographic symmetry, morphology, and size etc. During the transformation of the crystallographic symmetry (such as cubic $\rightarrow$ monoclinic), the crystallographic environment experiences a variation leading to the emission spectrum variation.

The crystal structure of $\mathrm{B}$-type $\mathrm{Eu}_{2} \mathrm{O}_{3}$ possesses $C 2 / m$ symmetry with $\mathrm{Eu}$ ions occupying three separate and distinct Wyckoff positions, each of which possess Cs symmetry. Within the resolution of these experiments, the emission lines could not be completely resolved. The luminescence spectrum obtained when releasing pressure to $0 \mathrm{GPa}$ is very similar to the profile in the luminescence spectrum of monoclinic $\mathrm{Eu}_{2} \mathrm{O}_{3}[37,38]$, but with an unresolved fine profile, which may result from the pressure effect. The luminescence spectrum obtained at $0 \mathrm{GPa}$ (decompression cycle) supports the speculation of a pressure-induced phase transition of A-type $\rightarrow$ B-type deduced from our XRD results. This reveals that the structural $\mathrm{C}$-type $\rightarrow$ A-type phase transition as the pressure decreases is irreversible. In addition, the relative intensity of luminescence of diamond compared to nano- $\mathrm{Eu}_{2} \mathrm{O}_{3}$ increases as the pressure decreases from $30 \mathrm{GPa}$ to $0 \mathrm{GPa}$.

\subsection{Impedance spectroscopy of nano- $\mathrm{Eu}_{2} \mathrm{O}_{3}$ under high pressure}

Under high pressure, a structural phase transition is often accompanied by an electronic phase transition. The decreasing atomic distance and variation of the crystal field of nano- $\mathrm{Eu}_{2} \mathrm{O}_{3}$ are expected to alter its electronic properties, which are reflected in the electronic transport property measurements. 
We now explore the effect of pressure on the transport behavior of nano- $\mathrm{Eu}_{2} \mathrm{O}_{3}$. The electronic configuration of $\mathrm{Eu}$ in $\mathrm{Eu}_{2} \mathrm{O}_{3}$ is $4 f^{6}\left(\mathrm{Eu}^{3+}\right)$, which belongs to the inner electron (subjected to shielding effect of the $5 s^{2} 5 p^{6}$ electrons) and characterized by its localized feature. Therefore, in general the rare earth oxides exhibit larger resistivity $\left(\sim 10^{9} \Omega \cdot \mathrm{cm}[39]\right.$, the resistivity of typical metal copper is $\sim 10^{-6}$ $\Omega \cdot \mathrm{cm})$. The resistivities of the rare earth sesquioxides become larger as the atomic number increase [40] according to reported literature results. The electron-electron interactions of $4 f$ electrons are strong and thus $\mathrm{Eu}_{2} \mathrm{O}_{3}$ is a strongly correlate system [41].

Impedance spectroscopy measurements on nano- $\mathrm{Eu}_{2} \mathrm{O}_{3}$ under high pressure demonstrated that the resistance of grains possesses a larger magnitude than the grain boundaries, and therefore one semicircle is seen clearly in the complex plane (inset of Fig. 6). The semicircle representing the transport properties of the grain boundaries cannot be observed. This may result from the comprehensive high pressure (compacting) and small particle size of our sample (nano size). From the Z'-intercept of semicircles, the resistance of grains $(R)$ at certain pressures can be deduced as shown in Fig. 6. The resistance decreases as the pressure increases from 0 to $12 \mathrm{GPa}$. A discontinuous change can be found at $\sim 12 \mathrm{GPa}$ as clearly seen in the inset of Fig. 6. These results show that the structural phase transition accompanies detectable changes in the electrical transport behavior. We attribute the unusual changes of resistance at $\sim 12 \mathrm{GPa}$ to the phase transitions of C-type $\rightarrow$ A-type. The change of the microstructure of grain boundaries (coming from different crystallographic phases, such as C-type and A-type $\mathrm{Eu}_{2} \mathrm{O}_{3}$ that coexist $\sim 12 \mathrm{GPa}$ ), affects the carrier scattering in the grain boundary conduction process. In our high pressure impedance spectrum experiment, we did not use a pressuretransmitting medium leading to a nonhydrostatic pressure environment in the sample. Compared with our XRD studies conducted under hydrostatic conditions, the transition pressure under non-hydrostatic compression has about a $3 \mathrm{GPa}$ shift to higher pressure. The anomalous variation $\sim 12 \mathrm{GPa}$ may occur for the following reasons: (i) The grain boundary microstructure rearranged during the structural phase 
transition. (ii) The electrons are subjected to stronger scattering from the A-type phase that emerged at $\sim 12 \mathrm{GPa}$ than the $\mathrm{C}$-type phase. As the pressure further increasing, the resistance decreases up to 22 $\mathrm{GPa}$. A one order of magnitude decrease in the resistance value was detected in the pressure range of 5 $\sim 20 \mathrm{GPa}$ by in-situ resistance measurements. We propose the nano- $\mathrm{Eu}_{2} \mathrm{O}_{3}$ compressed to $20 \mathrm{GPa}$ is an insulator from our impedance spectra measurements.

\subsection{The phase diagrams of the structural evolution process under high pressure for $\operatorname{Ln}_{2} \mathrm{O}_{3}$}

$\mathrm{Ln}_{2} \mathrm{O}_{3}$ exhibit interesting structural, physical and chemical properties and have important technological applications. Therefore, it is crucial to understand their structural stability at extreme conditions of pressure and temperature. During the past decades the rare earth sesquioxides have been studied by numerous workers who have determined the crystal structures and investigated the phase relationships among them. In order to systematically compare the structural evolution information on the compression behavior of $\mathrm{Ln}_{2} \mathrm{O}_{3}$, the structure phase diagrams of $\mathrm{Ln}_{2} \mathrm{O}_{3}$ under high pressure are reviewed and plotted in Fig. 7. The hashed bars extend upward to the highest pressure measurement made for each respective compounded. Since XRD, Raman spectroscopy and other techniques could characterize structural phase transitions, it is reminded that the pressure-induced structural phase transition in $\mathrm{Ln}_{2} \mathrm{O}_{3}$ summarized here are merely adapted to using XRD techniques. And the corresponding text descriptions abstracted from the phase diagram of $\mathrm{Ln}_{2} \mathrm{O}_{3}$ are as follows.

(I) For most of the $\mathrm{Ln}_{2} \mathrm{O}_{3}$, the B-type structure has been shown to be favored over the C-type, and the A-type over both of B- and C-types as the pressure increasing. However it is interesting to note that $\mathrm{La}_{2} \mathrm{O}_{3}$ and $\mathrm{Ce}_{2} \mathrm{O}_{3}$ were stable up to $18 \mathrm{GPa}$ and $70 \mathrm{GPa}$, respectively.

(II) It is found from Fig. 7 that the transition pressure (C-type $\rightarrow$ A-type, or B-type $\rightarrow$ A-type) is related to the atomic radii of lanthanides namely, and the transition pressure of $\mathrm{Ln}_{2} \mathrm{O}_{3}$ increases as the cationic radius decreases. 
(III) The C-type $\rightarrow$ A- and B-type phase transition possesses large coexistence pressure scope, while the B-type $\rightarrow$ A-type phase transition shows relatively clear phase boundary.

(IV) As Fig. 7 shows, for most of the $\mathrm{Ln}_{2} \mathrm{O}_{3}$, the C-type to B-type phase transition or to A-type have be observed as increasing pressure. While the full C-type $\rightarrow$ B-type $\rightarrow$ A-type structural phase transition sequence was only observed in $\mathrm{Sm}_{2} \mathrm{O}_{3}, \mathrm{Dy}_{2} \mathrm{O}_{3}$, and $\mathrm{Ho}_{2} \mathrm{O}_{3}$.

(V) For the light lanthanide sesquioxides such as $\mathrm{Sm}_{2} \mathrm{O}_{3}$ and $\mathrm{Eu}_{2} \mathrm{O}_{3}$, the C-type or B-type transformed into A-type as the pressure increases, and the high-pressure phase was unquenchable.

(VI) For the heavy lanthanide sesquioxides such as $\mathrm{Dy}_{2} \mathrm{O}_{3}, \mathrm{Ho}_{2} \mathrm{O}_{3}, \mathrm{Er}_{2} \mathrm{O}_{3}, \mathrm{Yb}_{2} \mathrm{O}_{3}$ and $\mathrm{Lu}_{2} \mathrm{O}_{3}$ and $\mathrm{Eu}_{2} \mathrm{O}_{3}$, the C-type transformed into B-type or/and A-type with pressure increasing, and the highpressure phase was quenchable. Though Gd belongs to heavy earth element, the structural evolution behavior for $\mathrm{Gd}_{2} \mathrm{O}_{3}$ is more similar with light rare earth sesquioxides.

\section{Conclusions}

To summarize, we found a structural phase transition in nano- $\mathrm{Eu}_{2} \mathrm{O}_{3}$ under high pressure through insitu high-pressure AD-XRD and Raman/luminescence spectroscopy measurements. A C-type $\rightarrow$ A-type phase transformation was observed in nano- $\mathrm{Eu}_{2} \mathrm{O}_{3} \sim 9 \mathrm{GPa}$. The A-type phase of nano- $\mathrm{Eu}_{2} \mathrm{O}_{3}$ was stable up to at least $43.0 \mathrm{GPa}$. Our reported nano- $\mathrm{Eu}_{2} \mathrm{O}_{3}$ transition pressure is higher than that of its corresponding bulk- $\mathrm{Eu}_{2} \mathrm{O}_{3}$. After decompression back to ambient pressure, the A-type phase was unquenchable and transformed into a $\mathrm{B}$-type phase. Compared with the bulk- $\mathrm{Eu}_{2} \mathrm{O}_{3}$, nano- $\mathrm{Eu}_{2} \mathrm{O}_{3}$ exhibited a larger bulk modulus. The structural phase transition observed from Raman and luminescence measurements were in good agreement with our XRD data analysis conclusion. The impedance spectrum measurement demonstrated that the anomaly; at $12 \mathrm{GPa}$ was likely due to the structural C-type $\rightarrow$ A-type phase transition. Furthermore, the general law and unique case for $\mathrm{Ln}_{2} \mathrm{O}_{3}$ 
under high pressure were clearly demonstrated by the summarized phase diagram of the structural evolution process for $\mathrm{Ln}_{2} \mathrm{O}_{3}$.

\section{Acknowledgements}

Portions of this work were performed at HPCAT (Sector 16), Advanced Photon Source (APS), Argonne National Laboratory. HPCAT is supported by CIW, CDAC, UNLV, and LLNL through funding from DOE-NNSA, DOE-BES, and NSF. Use of the Advanced Photon Source, an Office of Science User Facility operated for the US DOE Office of Science by Argonne National Laboratory, was supported by the US DOE under Contract No. DE-AC02-06CH11357. The authors acknowledge the support of NSAF (Grant No: U1530402) and Science Challenging Program. This work was supported by the Program for New Century Excellent Talents in University (NCET-10-0444) project by Professor Lin Wang. 


\section{REFERENCES}

1. M. Zinkevich, Prog. Mater. Sci. 52 (2007) 597.

2. $\quad$ M. Tang, J. A. Valdez, K. E. Sickafus, P. Lu, Appl. Phys. Lett. 90 (2007) 151907.

3. L. Kittiratanawasin, R. Smith, B. P. Uberuaga, K. E. Sickafus, J. Phys. Condens. Matter 21 (2009) 115403.

4. $\quad$ G. Y. Adachi, N. Imanaka, Chem. Rev. 98 (1998) 1479.

5. $\quad$ S. Jiang, J. Liu, C. L. Lin, X. D. Li, and Y. C. Li, J. Appl. Phys. 113 (2013) 113502.

6. F. X. Zhang, M. Lang, J. W. Wang, U. Becker, and R. C. Ewing, Phys. Rev. B 78 (2008) 064114.

7. S. Jiang, J. Liu, C. L. Lin, L. G. Bai, Y. F. Zhang, X. D. Li, Y. C. Li, L. Y. Tang, H. Wang, Solid State Commun. 169 (2013) 37.

8. H. Yusa, T. Tsuchiya, N. Sata, and Y. Ohishi, Inorg. Chem. 48 (2009) 7537.

9. H. Yusa, T. Tsuchiya, N. Sata, and Y. Ohishi, Inorg. Chem. 49 (2010) 4478.

10. D. Richard, L. A. Errico, M. Rentería, J. Alloy. Compd. 664 (2016) 580.

11. G. Chen, N. A. Stump, R. G. Haire, and J. R. Peterson, J. Alloy. Compd. 181 (1992) 503.

12. G. Chen, J. R. Peterson, and K. E. Brister, J. Solid State Chem. 111 (1994) 437.

13. S. Jiang, L. G. Bai, J. Liu, W. S. Xiao, X. D. Li, Y. C. Li, L.Y. Tang, Y. F. Zhang, and D. C. Zhang, Chin. Phys. Lett. 26 (2009) 076101.

14. Z. W. Wang, L. L. Daemen, Y. S. Zhao, C. S. Zha, R. T. Downs, X. D. Wang, Z. L. Wang, and R. J. Hemley, Nat. Mater. 4 (2005) 922.

15. R. S. Kumar, A. L. Cornelius, M. F. Nicol, Curr. Appl. Phys. 7 (2007) 135.

16. L. Wang, W. G. Yang, Y. Ding, Y. Ren, S. G. Xiao, B. B. Liu, S. V. Sinogeikin, Y. Meng, D. J. Gosztola, G. Y. Shen, R. J. Hemley, W. L. Mao, and H. K. Mao, Phys. Rev. Lett. 105 (2010) 095701 .

17. Z. W. Wang, S. K. Saxena, V. Pischedda, H. P. Liermann, and C. S. Zha, Phys. Rev. B 64 (2001) 012102.

18. J. S. Olsen, L. Gerward, and J. Z. Jiang, J. Phys. Chem Solids 60 (1999) 229.

19. J. Z. Jiang, J. S. Olsen, L. Gerward, and S. Morup, Europhys. Lett. 44 (1998) 620.

20. N. Dilawar, D. Varandani, V. P. Pandey, M. Kumar, S. M. Shivaprasad, P. K. Sharma, and A. K. Bandyopadhyay, J. Nanosci Nanotechno. 6 (2006) 105.

21. A. Hammersley, S. Svensson, M. Hanfland, A. Fitch and D. Hausermann, High Pressure Res. 14 (1996) 235.

22. H. K. Mao, J. Xu, P. Bell, J. Geophys. Res. 91 (1986) 4673.

23. M. Xu, Z. H. Yu, L. Wang, R. Mazzarello, and M. Wuttig, Adv. Electron. Mater. 1 (2015) 1500240.

24. A. C. Larson, R. B. Von Dreele, General Structure Analysis System (GSAS), Los Alamos National Laboratory Report LAUR 86-748, 1994.

25. B. H. Toby, J. Appl. Crystallorg. 34 (2001) 210.

26. S. B. Qadri, J. Yang, B. R. Ratna, E. F. Skelton, and J. Z. Hu, Appl. Phys. Lett. 69 (1996) 2205.

27. J. Z. Jiang, J. S. Olsen, L. Gerward, D. Frost, D. Rubie, and J. Peyronneau, Europhys. Lett. 50 (2000) 48.

28. $\quad$ R. J. Angel, J. Gonzalez-Platas, and M. Alvaro, Z. Kristallogr. 229 (2014) 405.

29. F. Birch, Phys. Rev. 71 (1947) 809.

30. G. Concas, J. K. Dewhurst, A. Sanna, S. Sharma, and S. Massidda, Phys. Rev. B 84 (2011) 014427.

31. W. B. White, V. G. Keramidas, Spectrochim. Acta, Part A 28 (1972) 501.

32. L. A. Tucker, F. J. Carney, P. McMillan, S. H. Lin, and L. Eyring, Appl. Spectrosc. 38 (1984) 857. 
33. N. Dilawar, D. Varandani, S. Mehrotra, H. K. Poswal, S. M. Sharma and A. K. Bandyopadhyay, Nanotechnology 19 (2008) 115703.

34. A. V. Prokofiev, A. I. Shelykh, B. T. Melekh, J. Alloys Compd. 242 (1996) 41.

35. K. C. Sheng, G. M. Korenowski, J. Phys. Chem. 92 (1988) 50.

36. $\quad$ F. Occelli, P. Loubeyre, R. LeToullec, Nature materials 2 (2003) 151.

37. B. Bihari, H. Eilers, B. M. Tissue, J. Lumin. 75 (1997) 1.

38. J. Dexpert-Ghys, M. Faucher, and P. Caro, Phys. Rev. B 23 (1981) 607.

39. V. M. Koleshko, N. V. Babushkina, Thin Solid Films, 62 (1979) 1.

40. G. V. Subba Rao, S. Ramdas, P. N. Mehrotra, C. N. R. Rao, J. Solid State Chem. 2 (1970) 377.

41. R. Gillen, S. J. Clark, and J. Robertson, Phys. Rev. B 87 (2013) 125116.

42. S. Jiang, J. Liu, C. L. Lin, L. G. Bai, W. S. Xiao, Y. F. Zhang, D. C. Zhang, X. D. Li, Y. C. Li, and L. Y. Tang, J. Appl. Phys. 108 (2010) 083541.

43. C. M. Lin, K. T. Wu, T. L. Hung, H. S. Sheu, M. H. Tsai, J. F. Lee, J. J. Lee, Solid State Commun. 150 (2010) 1564.

44. C. Meyer, J. P. Sanchez, J. Thomasson, and J. P. Itié, Phys. Rev. B 51 (1995) 12187.

45. S. D. Pandey, K. Samanta, J. Singh, N. D. Sharma, and A. K. Bandyopadhyay, AIP Advances 3 (2013) 122123.

46. P. Ch. Sahu, D. Lonappan and N. V. Chandra Shekar, J. Phys. Conf. Ser. 377 (2012) 012015.

47. X. T. Ren, X. Z. Yan, P. Wang, Y. Li, S. M. Wang, F. Peng, L. Xiong, J. Liu \& D. W. He, High Press Res. 34 (2014) 70.

48. Q. X. Guo, Y. S. Zhao, C. Jiang, W. L. Mao, Z. W. Wang, J. Z. Zhang, and Y. J. Wang, Inorg. Chem. 46 (2007) 6164.

49. S. Jiang, J. Liu, X. D. Li, L. G. Bai, W. S. Xiao, Y. F. Zhang, C. L. Lin, Y. C. Li, and L. Y. Tang, J. Appl. Phys. 110 (2011) 013526.

50. D. Lonappan, N. V. Chandra Shekar, T. R. Ravindran, P. Ch. Sahu, Mater. Chem. Phys. 120 (2010) 65.

51. L. G. Bai, J. Liu, X. D. Li, S. Jiang, W. S. Xiao, Y. C. Li, L. Y. Tang, Y. F. Zhang, and D. C. Zhang, J. Appl. Phys. 106 (2009) 073507.

52. Q. X. Guo, Y. S. Zhao, C. Jiang, W. L. Mao, Z. W. Wang, Solid State Commun.145 (2008) 250.

53. M. J. Lipp, J. R. Jeffries, H. Cynn, J.-H. Park Klepeis, W. J. Evans, D. R. Mortensen, G. T. Seidler, Y. Xiao, and P. Chow, Phys. Rev. B 93 (2016) 064106.

54. J. P. McClure, PhD thesis, University of Nevada, Las Vegas (2009). 


\section{Figure captions}

Fig. 1. Schematic crystal structures of polymorphism for $\mathrm{Eu}_{2} \mathrm{O}_{3}$, (a) C-type, space group: Ia-3 (No. 206), A-type, space group: $P-3 m$ (No. 164), and B-type space group: $C 2 / m$ (No. 12). (b) TEM image of nano- $\mathrm{Eu}_{2} \mathrm{O}_{3}$. The scale bar is also presented for convenient estimation of the sample particle size.

Fig. 2 (a). AD-XRD pattern of nano- $\mathrm{Eu}_{2} \mathrm{O}_{3}$ under various pressures up to $43.0 \mathrm{GPa}$ at room temperature during the compression process. (b) The XRD pattern collected in the releasing pressure process.

Fig. 3 (a). Pressure dependence of the normalized lattice parameters for $\mathrm{C}-$ and $\mathrm{A}$-type nano- $\mathrm{Eu}_{2} \mathrm{O}_{3}$. The inset shows the axial ratio for the A-type phase as a function of pressure. The axial compressibilities of the A-type phase are remarkably anisotropic; the compressibility along the $a$-axis is about one order of magnitude smaller than the $c$-axis. (b) Pressure-dependent volume of nano- $\mathrm{Eu}_{2} \mathrm{O}_{3}$ (this work) and previous data from Jiang et al. (bulk $\mathrm{Eu}_{2} \mathrm{O}_{3}$, Ref. [13]). The bulk moduli of the C-type and A-type phases of bulk- and nano- $\mathrm{Eu}_{2} \mathrm{O}_{3}$ are presented for convenient comparison.

Fig. 4. Raman spectra of nano- $\mathrm{Eu}_{2} \mathrm{O}_{3}$ under various pressures at ambient temperature. Top panel, the Raman spectrum collected after sample release to ambient conditions is marked with R0 GPa.

Fig. 5 (a). Selected luminescence $\left({ }^{5} \mathrm{D}_{0} \rightarrow{ }^{7} \mathrm{~F}_{0}, \sim 580 \mathrm{~nm}\right)$ spectra of nano-Eu $\mathrm{E}_{2} \mathrm{O}_{3}$ under different pressures. The luminescence spectrum obtained under releasing pressure to ambient pressure is marked with R0. The luminescence from diamond is also labeled with dashed lines. (b) The relative loss of intensity in band I with respect to band II under high pressure.

Fig. 6. The pressure dependence of nano- $\mathrm{Eu}_{2} \mathrm{O}_{3}$ resistance; an anomalous variation was observed at $\sim$ $12 \mathrm{GPa}$. The inset shows the impedance spectra (in the complex plane) of nano- $\mathrm{Eu}_{2} \mathrm{O}_{3}$ under different pressures. The solid lines in the inset are a visual aid.

Fig. 7. The phase diagrams of Lanthanide sesquioxides under room temperature with increasing pressure referred from several adapted literatures (Ref. 5-7, 12, 13, 20, 42-54) and present work. The data plotted in this figure was merely carried out using experimental XRD techniques. The right edge of the bars indicates the maximum experimental pressure. The sample morphology was marked with "Bulk", "Submicron", and "Nano". 


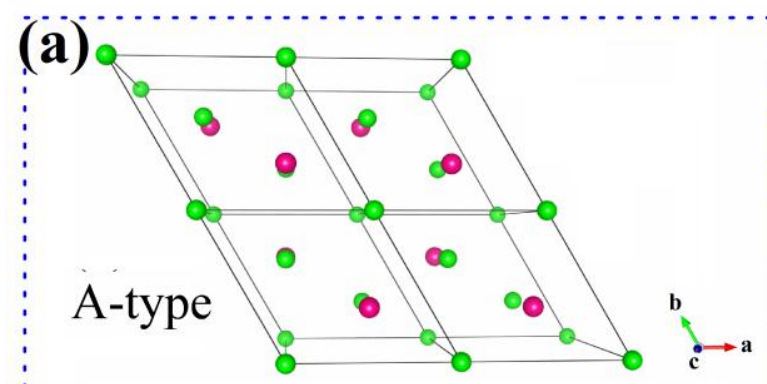

(b)

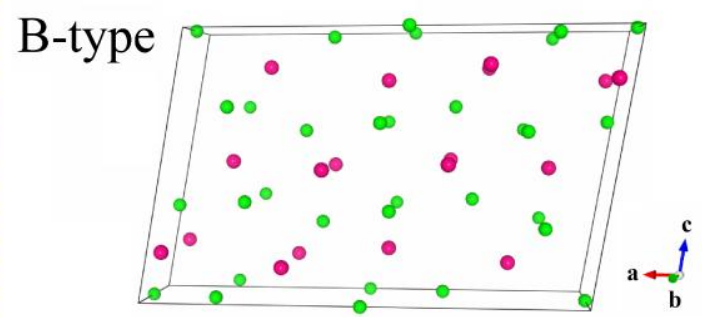

C-type
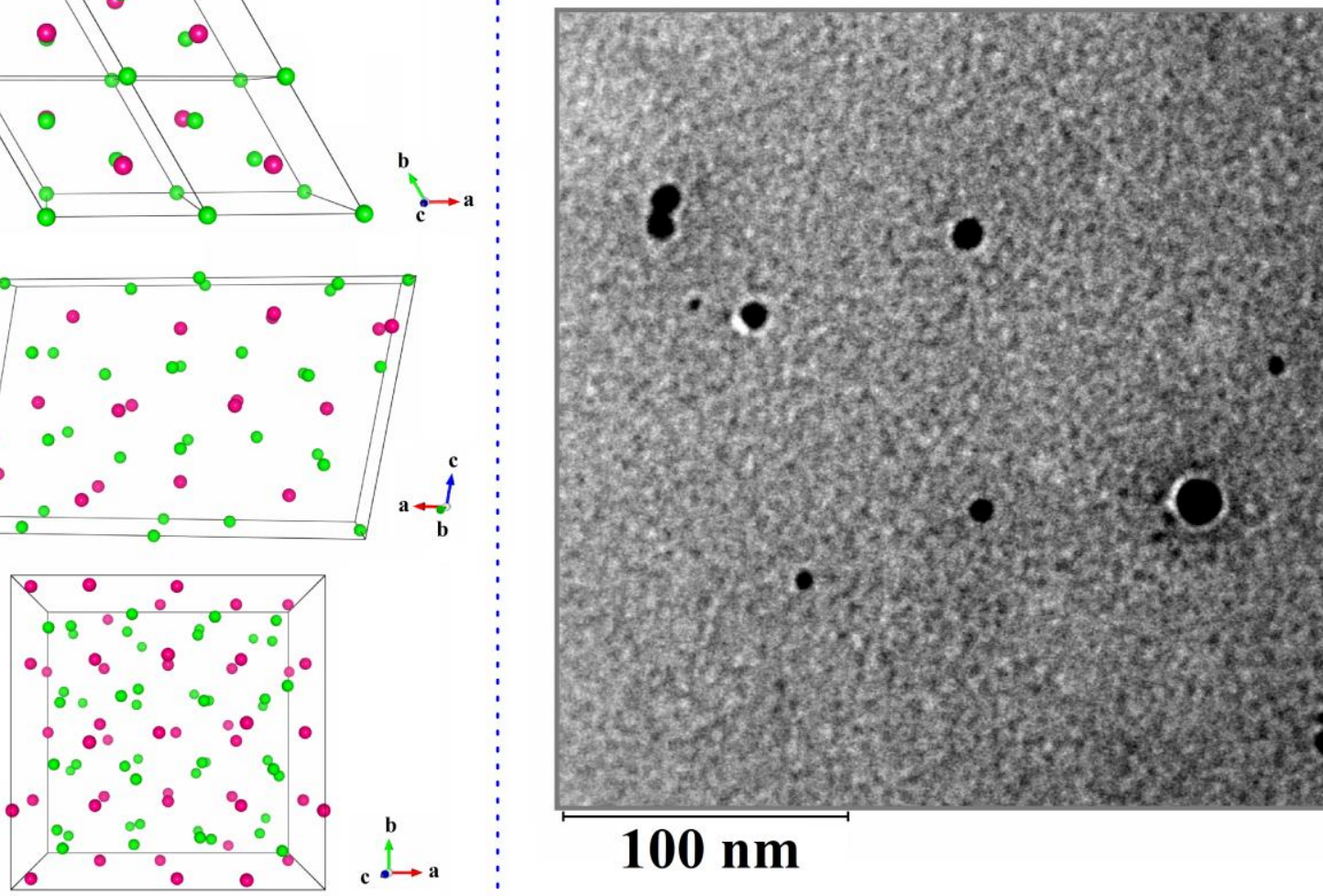

$100 \mathrm{~nm}$ 

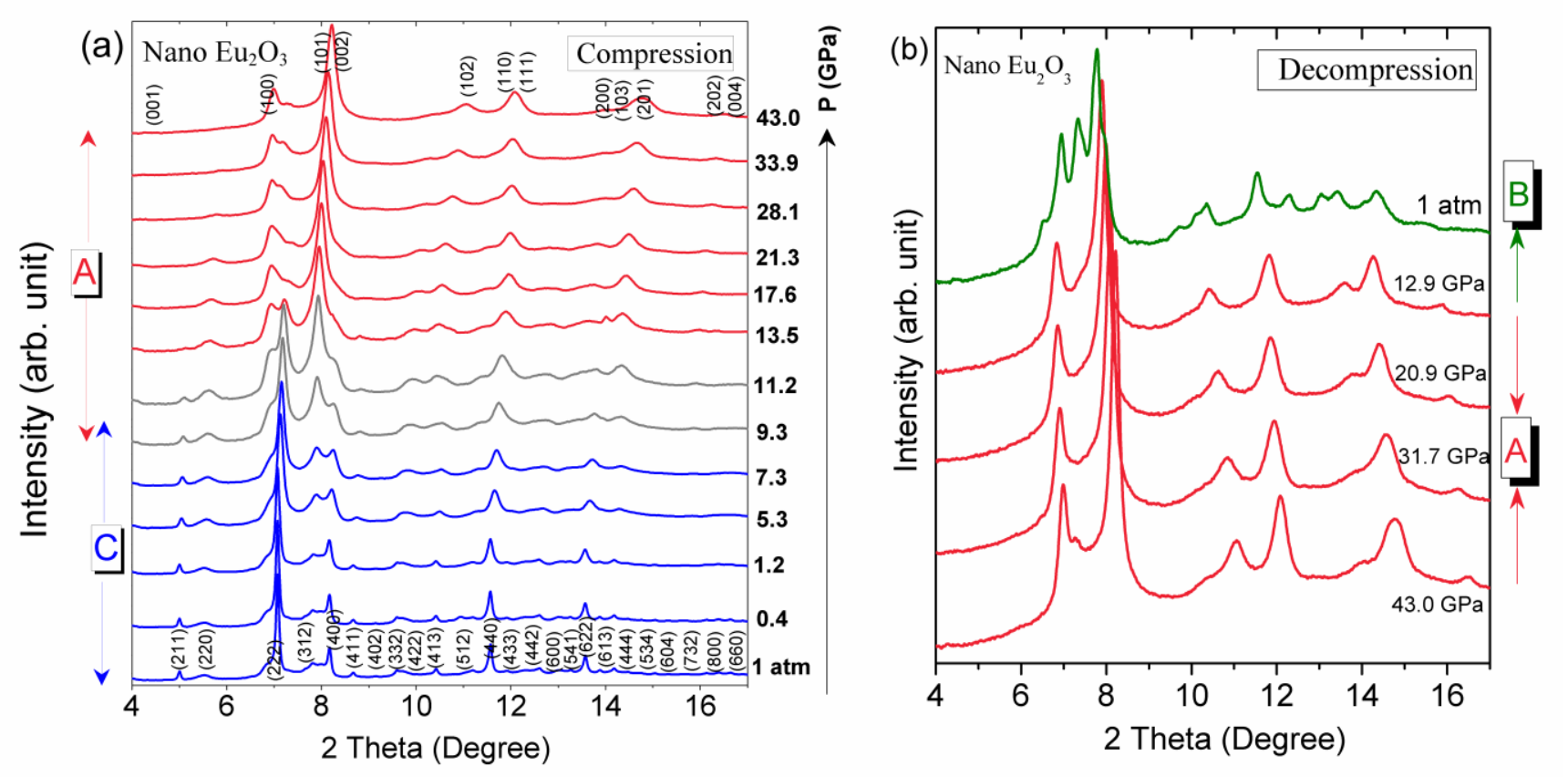

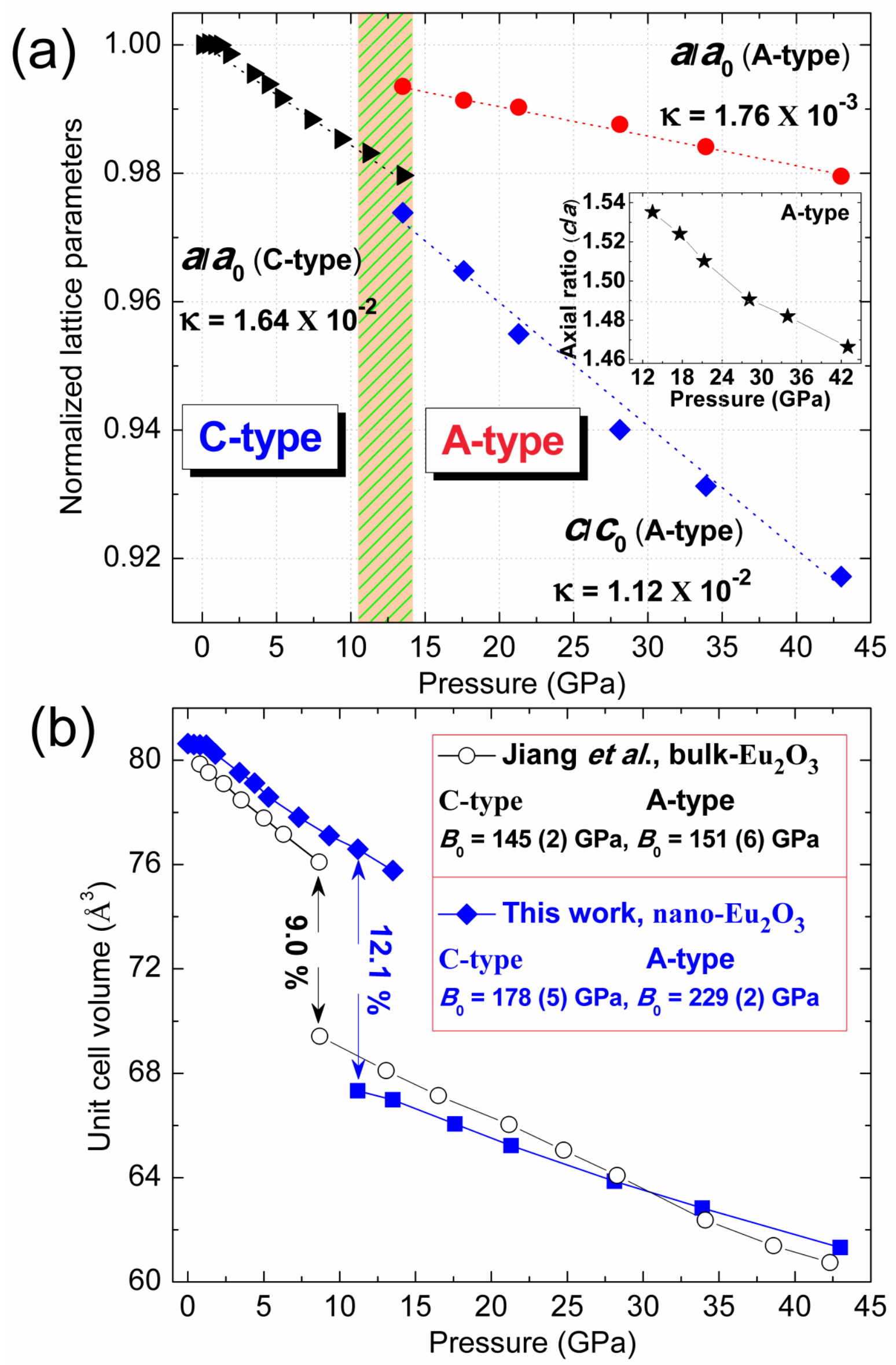


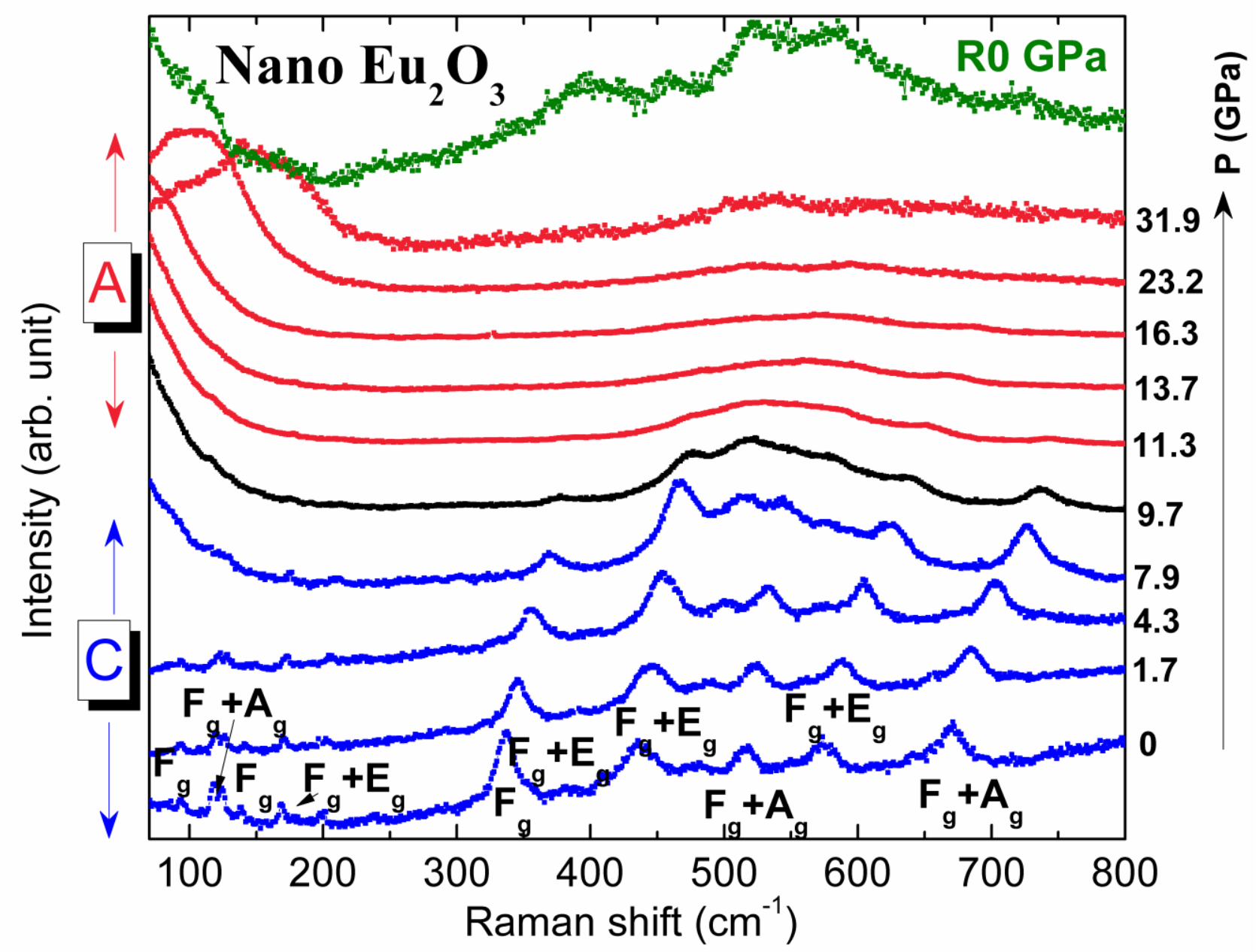



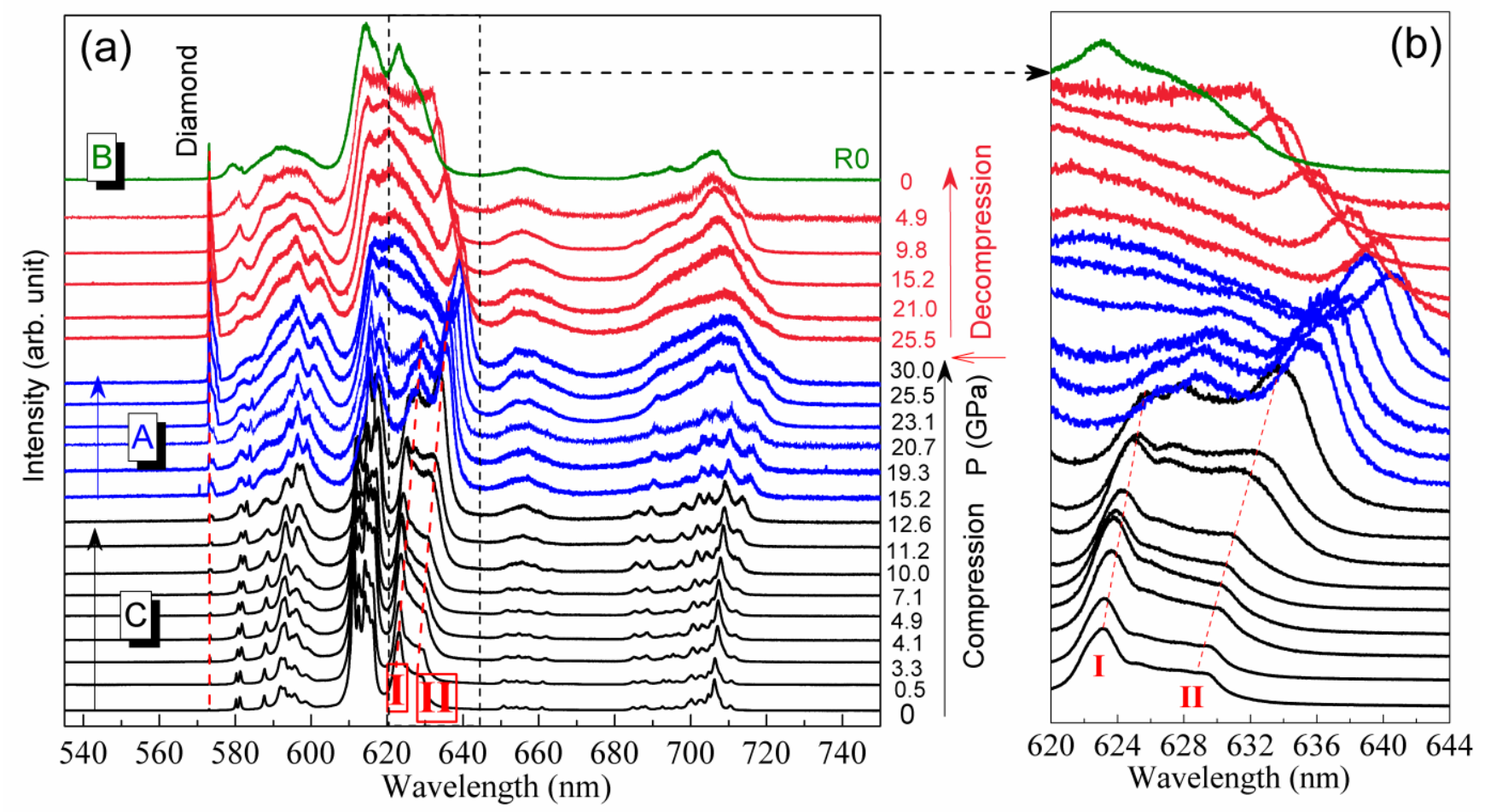


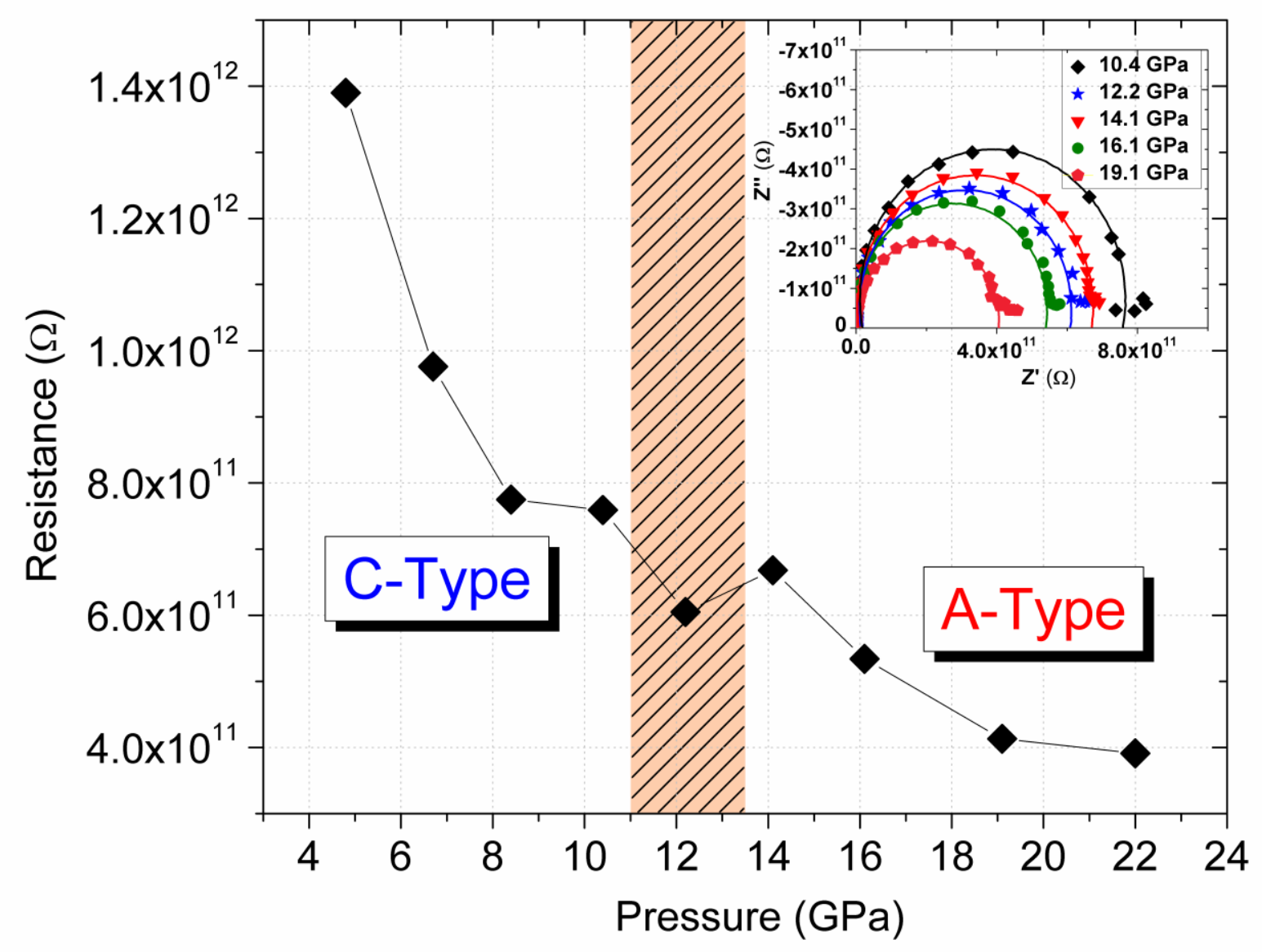




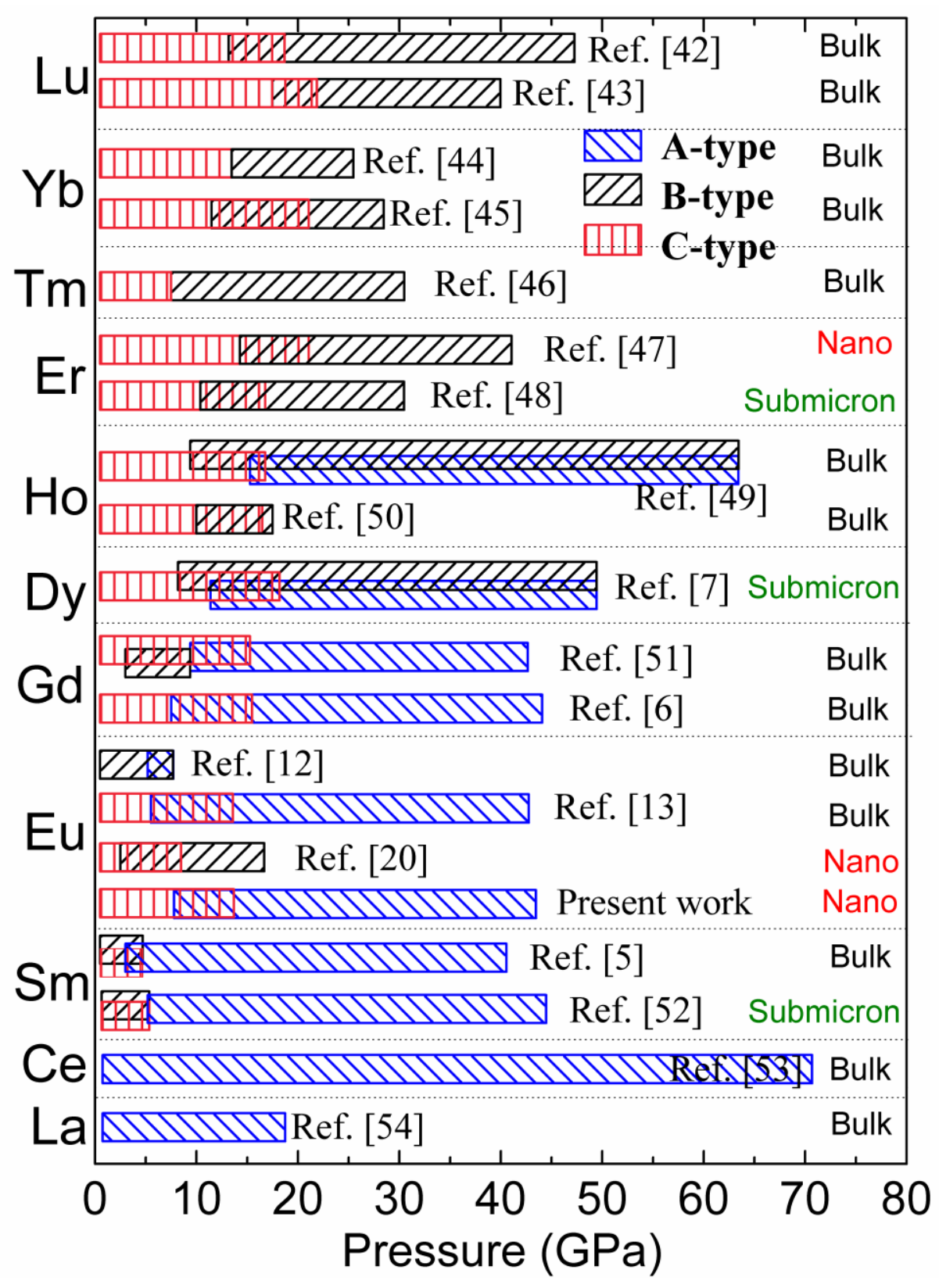

